\title{
Optimization of Resource Allocation in Multihop HARQ Relay Networks with a Delay Constraint
}

\author{
Jun-Mei HAN ${ }^{a}$, Xu LIU, Yong XI and Ji-Bo WEI \\ School of Electronic Science and Computer Engineering, National University of Defence Technology, Changsha, \\ 410073 China
}

\begin{abstract}
By minimizing the outage probability, optimization is carried out in this paper to find joint optimal power allocation (OPA) and relay placement (ORP) for multihop relay networks adopting Hybrid Automatic Repeat reQuest (HARQ). Different from previous works, the joint OPA and ORP is analysed under generalized fading channels with the constraint on total transmit power, end-to-end relaying distance and maximum transmission number (delay). The simulation results demonstrate that for different fixed number of nodes and fading models, there are preferred deployments depending on path loss exponent and power retransmission strategy. By employing multiple retransmission round which can improve the reliability and energy efficiency without significant overhead, the end-to-end outage probability is no longer bounded by that of the weaker hop, i.e., the hop with a poor channel condition. The proposed strategy provides a dramatic improvement for the end-to-end outage probability by compensating the channel difference.
\end{abstract}

\section{Introduction}

Multihop Hybrid-automatic repeat request (HARQ) relay networks, which combined the advantage of multihop relay and HARQ techniques, have great potential to improve the performance of wireless networks due to their ability to improve the reliability and efficiency of transmission. In particular, the presence of multiple users between source and destination enables the division of long links into multiple shorter links which reduce signal attenuation, extends battery life, and provides broader and cheaper coverage along with high efficiency. On the other hand, HARQ protocols are widely employed to increase the reliability of packet transmissions by combining forward error-correcting coding (FEC) technique with ARQ technique. Because multiple retransmission rounds can improve the transmission reliability and efficiency without significant overhead, the performance of multihop relay networks can be improved by adopting HARQ schemes. Although various schemes have been proposed to analyze the performance of multihop relay networks and HARQ schemes, optimization of these two techniques are usually investigated separately.

The end-to-end (e2e) performances of the multihop relay networks have been extensively studied in the literature where most of them consider only one transmission round. In particular, the joint optimization of power allocation (OPA) and relay placement (ORP) is presented for multihop communication in a Rayleigh-fading environment in [1]. The distance-dependent route selection

\footnotetext{
${ }^{a}$ Corresponding author: hjm.han@163.com
} 
problem is approached from the viewpoint of energy efficiency in [2]. By minimizing the outage probability of a decode-and-forward (DF) MIMO relay system with orthogonal space-time block coding, [3] shows that relay position optimization improves the finite-SNR (signal-to-noise ratio) diversity gain of a relay system whose adjacent hops have different diversity orders (unbalanced system). These studies show that joint optimization outperforms separate optimization significantly by improving the coding gain and diversity order.

By assuming fixed relay placement, optimal rate and power allocation problems have been addressed for truncated HARQ based multihop systems in several literatures. For instance, [4] proposed a joint power and rate optimization scheme to maximize the long-term average transmission rate of the multihop HARQ relay networks under Rayleigh-fading channels. Likewise, the e2e outage probability was studied in [5] by using a Markov chain model. However, these studies are limited to the Rayleigh-fading channels and have only analysed the e2e performance without attempting to optimize the system parameters for further performance enhancement.

In this paper, by modeling the retransmission number of a packet from source to destination as delay, the e2e outage probability is derived under generalized fading channels and will be employed as the performance metric in the system optimization. For the power and delay limited applications, we consider the following two constraints: 1) The maximum retransmission number should no more than $L ; 2)$ The sum of the power for all retransmission rounds for one packet should be less than or equal to $P_{T}$. We focus on two issues in multihop HARQ relay networks, i.e., how to allocate power and relay position for each relay and how to allocate retransmission times for each hop to minimize the outage probability under power consumption constraint. Note that the two issues are closely correlated and should be jointly optimized. Moreover, due to the versatile and flexible features of generalized fading, it is worthy to note that the generalized fading channel has the advantage of including various fading models such as Hoyt, Nakagami- $m$, and Rician fading as special cases [6].

\section{System model and outage probability}

We consider a linear $M$-hop network consisting of $M+1$ nodes under block-fading channel, where relays are serially connected from the source to the destination and the channel gain is constant during a single HARQ round, but the channel gains of different HARQ rounds are independent and identically distributed (i.i.d.). Each node has a single antenna and uses half-duplex transmission and performs with maximal-ratio combining (MRC). For different hops $R_{i} \neq R_{j}$, the channel gains $h_{i}$ and $h_{j}$ are mutually independent and may assume different fading models. We use the fixed and normalized source-to-destination distance $\left(d_{S D}=\sum_{m=1}^{M} d_{m}=1,0<d_{m}<1\right)$, where $d_{m}$ is the distance between any two terminals. Without loss of generality, we assume $\varepsilon\left[\left|h_{m}\right|^{2}\right]=d_{m}^{-\alpha}$, where $\varepsilon[\cdot]$ denotes the expectation and $\alpha$ is the path-loss exponent. Assuming that the transmission power keeps constant in each (re)transmission round ( i.e., $P_{l_{i}}=P_{m}$ for $i=1,2, \ldots, m$.), SNR of the i-th hop is denoted as $\gamma_{m}=\left(P_{m} / N_{0}\right)\left|h_{m}\right|^{2}$ with $\varepsilon\left[\gamma_{m}\right]=\bar{\gamma}_{m}=P_{m} /\left(N_{0} d_{m}^{\alpha}\right)$, where $N_{0}$ denotes the AWGN power spectral density.

We first analysis the approximate outage probability for the $m$-th hop after $l_{m}$ retransmission with the moment generating function (MGF)-based approach [6]. By adopting the results in [7], the probability density function (PDF) of $\bar{\gamma}_{m}$ is approximated by:

$$
f_{\gamma_{m}}(x) \approx \frac{a_{m}}{t_{m}}\left(\frac{x}{\bar{\gamma}_{m}}\right)^{t_{m}}
$$


where $t_{m}$ is related to the diversity order of the channel, and $a_{m}$ is related to the SNR gain of the i-th hop. The parameter pairs $\left\{t_{m}, a_{m}\right\}$ for Nakagami- $m$, Rician, and Hoyt fading channels are provided in Table I of [8]. Accordingly, the MGF of $\gamma_{m}$ can be written as:

$$
\mathrm{M}_{\gamma_{m}}(s)=\int_{0}^{\infty} f_{\gamma_{m}}(x) e^{-s x} d x=\frac{a_{m}}{t_{m}}\left(\frac{1}{\bar{\gamma}_{m}}\right)^{t_{m}}\left(\frac{\Gamma\left(1+t_{m}\right)}{s^{1+t_{m}}}\right)
$$

where $\Gamma(m)=\int_{0}^{\infty} t^{m-1} e^{-t} d t$ is the Gamma function. We define $Z \triangleq \sum_{i=1}^{l_{m}} \gamma_{i}$, then the MGF of $Z$ is given by: $\mathrm{M}_{Z}(s)=\left(a_{m} \Gamma\left(1+t_{m}\right) /\left(t_{m} \bar{\gamma}_{m}^{t_{m}} s^{t_{m}+1}\right)\right)^{l_{m}}$ The PDF of $Z$ can be written as :

$$
f_{Z}(z)=L^{-1}\left(\mathrm{M}_{Z}(s)\right)=\left(\frac{a_{m} \Gamma\left(1+t_{m}\right)}{t_{m} \bar{\gamma}_{m}^{t_{m}}}\right)^{l_{m}} \frac{z^{\left(1+t_{m}\right) l_{m}-1}}{\Gamma\left(l_{m}+l_{m} t_{m}\right)}
$$

Accordingly, the approximate outage probability of the $m$ th hop can be calculated as

$$
\operatorname{Pr}\left\{\sum_{i=1}^{l_{m}} \bar{\gamma}_{m}<\gamma_{t h}\right\}=F_{Z}\left(\gamma_{t h}\right)=\int_{0}^{\gamma_{t h}} f_{Z}(t) d t=\left(\frac{a_{m} \Gamma\left(1+t_{m}\right)}{t_{m} \bar{\gamma}_{m}^{t_{m}}}\right)^{l_{m}} \frac{\gamma_{t h}^{l_{m}\left(1+t_{m}\right)}}{l_{m}\left(1+t_{m}\right) \Gamma\left(l_{m}+l_{m} t_{m}\right)}
$$

where $F_{Z}$ is the cumulative distribution function (CDF) of $Z, \gamma_{t h}$ is the SNR threshold and $l_{m}$ is the maximum retransmission number for $m$ th hop.

Now we investigate the multihop case. The e2e outage probability of multihop HARQ relay network is given by

$$
P_{e 2 e}=\operatorname{Pr}\left\{\min \left(\sum_{l_{1}=1}^{l_{1}} \gamma_{l_{1}}, \sum_{l_{2}=1}^{l_{2}} \gamma_{l_{2}}, \ldots, \sum_{l_{m}=1}^{l_{m}} \gamma_{l_{m}}\right) \leq \gamma_{t h}\right\}=1-\prod_{m=1}^{M} \operatorname{Pr}\left\{\gamma_{m}>\gamma_{t h}\right\} \approx \sum_{i=1}^{M} F_{\gamma_{i}}\left(\gamma_{t h}\right)
$$

Since the integration term is much smaller than the sum term, we ignore it approximately. Thus, the e2e outage probability is given by:

$$
P_{e 2 e} \approx \sum_{m=1}^{M} \operatorname{Pr}\left\{\sum_{i=1}^{l_{m}} \bar{\gamma}_{m}<\gamma_{t h}\right\}=\sum_{m=1}^{M}\left(\frac{a_{m} \gamma_{t h}^{\left(1+t_{m}\right)} N_{0}^{t_{m}} \Gamma\left(1+t_{m}\right)}{t_{m} l_{m}\left(1+t_{m}\right) \Gamma\left(l_{m}+l_{m} t_{m}\right)}\left(\frac{d_{m}^{\alpha t_{m}}}{P_{m}^{t_{m}}}\right)\right)^{l_{m}}
$$

In the following, by assuming the retransmission number of each hop to be an constant, we first formulate the joint optimizations of power allocation $\mathbf{P}=\left[P_{1}, P_{2}, \ldots, P_{N}\right]$ and relay placement $\mathbf{d}=\left[d_{1}, d_{2}, \ldots, d_{N}\right]$ which minimizes $P_{e 2 e}$ in (5). Then, the retransmission allocation strategy are studied based on the joint OPA and ORP optimization strategy.

\section{Joint optimization of PA and RP}

With the definition of the e2e outage probability, the joint OPA and ORP optimization problem is given by: 


$$
\begin{aligned}
& \min P_{e 2 e}=\sum_{m=1}^{M} \mathrm{~F}(\mathbf{P}, \mathbf{d}) \\
& \text { s.t. } \sum_{m=1}^{M} P_{m} l_{m} \leq P_{T} ; \sum_{m=1}^{M} l_{m}=L \text { and } \sum_{m=1}^{M} d_{m}=1
\end{aligned}
$$

where $\mathrm{F}(\mathbf{P}, \mathbf{d})=\mathrm{A}(m)\left(d_{m}^{\alpha} P_{m}^{-1}\right)^{l_{m} t_{m}}$ and $\mathrm{A}(m)=\left(\frac{a_{m} \gamma_{t h}^{\left(1+t_{m}\right)} N_{0}^{t_{m}} \Gamma\left(1+t_{m}\right)}{t_{m} l_{m}\left(1+t_{m}\right) \Gamma\left(l_{m}+l_{m} t_{m}\right)}\right)^{l_{m}}$. The optimization problem in (7) is not always convex with respect to the optimization variables. In particular, the nonconvexity arises from the objective function and is related to the distance $d_{m}$, the retransmission round $l_{m}$ and the pathloss factor $\alpha$. In order to obtain tractable solution for the optimization problem, we first recast optimization problem (7) as a convex optimization problem and then study the relationship of $d_{m}, l_{m}$ and $\alpha$.

Theorem 1: The problem in (7) is a convex optimization problem if $\alpha\left(t_{m} l_{m}(\alpha-1)-1\right)>0$ for $m=1,2, \ldots, M$.

Proof: Taking the first-order derivative of function $\mathrm{F}(\mathbf{P}, \mathbf{d})$ with respect to $P_{m}$, we can obtain that $\frac{\partial \mathrm{F}}{\partial P_{m}}=-\frac{t_{m} l_{m} d_{m m_{m} t_{m}} \mathrm{~A}(m)}{P_{m}^{t_{m} l_{m}+1}}$ Then we check the second-order derivative of function $\mathrm{F}(\mathbf{P}, \mathbf{d})$ with respect to $P_{m} \cdot \frac{\partial^{2} \mathrm{~F}}{\partial P_{m}^{2}}=\frac{t_{m} l_{m} d_{m}^{\alpha l_{l_{m} t_{m}}}\left(t_{m_{m}} l_{m}+1\right) \mathrm{A}(m)}{P_{m}^{t_{m} l_{m}+2}}$. Similarly, we can get the first-order and second-order derivatives of function $\mathrm{F}(P, d)$ with respect to $d_{m}$ as $\frac{\partial \mathrm{F}}{\partial d_{m}}=\frac{\alpha t_{m} l_{m} d_{m}^{\alpha_{m} l_{m} t_{m}-1} \mathrm{~A}(m)}{P_{m}^{t_{m} l_{m}}}$ and $\frac{\partial^{2} \mathrm{~F}}{\partial d_{m}^{2}}=\frac{\alpha t_{m} l_{m}\left(\alpha t_{m} l_{m}-1\right) d_{m}^{\alpha_{m} l_{m} t_{m}-2} \mathrm{~A}(m)}{P_{m}^{t_{m} l_{m}}}$, respectively.

The Hessian matrix of $\mathrm{F}$ is given by

$$
H(F)=\left[\begin{array}{cc}
\frac{\partial^{2} \mathrm{~F}}{\partial P_{m}^{2}} & \frac{\partial^{2} \mathrm{~F}}{\partial P_{m} \partial d_{m}} \\
\frac{\partial^{2} \mathrm{~F}}{\partial d_{m} \partial P_{m}} & \frac{\partial^{2} \mathrm{~F}}{\partial d_{m}^{2}}
\end{array}\right]
$$

where $\frac{\partial^{2} \mathrm{~F}}{\partial d_{m} \partial P_{m}}=\frac{\partial^{2} \mathrm{~F}}{\partial P_{m} \partial d_{m}}=-\frac{\alpha\left(t_{m} l_{m}\right)^{2} d_{m}^{\alpha_{m} l_{m} t_{m}-1} A(m)}{P_{m}^{t_{m} l_{m}+1}}$ The determinant of the Hessian matrix can be evaluated as :

$$
\operatorname{det}[H(\mathrm{~F})]=\alpha\left(t_{m} l_{m}(\alpha-1)-1\right)\left(\frac{d_{m}^{\alpha l_{m} t_{m}-2} t_{m} l_{m} A(m)}{P_{m}^{t_{m} l_{m}+2}}\right)^{2}
$$

Obviously, if $\alpha\left(t_{m} l_{m}(\alpha-1)-1\right)>0$, then (9) is always positive, which is equivalent to that $\mathrm{F}$ is jointly convex. Since the constraints are linear and the non-negative summation of convex functions is convex, Theorem 1 is proved.

Note that $l_{m}>1$ and $\alpha>0$ always hold true, moreover, $\alpha$ is normally in the range of 2-6 and $t_{m}$ is normally no less than 1 [6], accordingly, $\alpha\left(t_{m} l_{m}(\alpha-1)-1\right)>0$ holds true for most typical fading channel models. In the rare case, i.e., in buildings and stores when $\alpha\left(t_{m} l_{m}(\alpha-1)-1\right)<0$, geometric programming (GP) approximation [9] can be adopt to obtain the solution. The parameters for the approximation are similar with that in [8]. 
To solve this problem, we introduce nonzero Lagrange multipliers $\lambda$ and $\mu$, the Lagrange function for joint optimization problem of PA and RP can be formulated as:

$$
\Gamma=\sum_{m=1}^{M} A(m)\left(\frac{d_{m}^{\alpha}}{P_{m}}\right)^{l_{m} t_{m}}+\lambda\left(\sum_{m=1}^{M} l_{m} P_{m}-P_{T}\right)+\mu\left(\sum_{m=1}^{M} d_{m}-1\right)
$$

Take the first derivatives of $\mathrm{F}(\mathbf{P}, \mathbf{d})$ with respect to $P_{m}$ and $d_{m}$ to zero, we can obtain the optimal solution $(\mathbf{P}, \mathbf{d})$ :

$$
\begin{gathered}
\frac{\partial \Gamma}{\partial P_{m}}=-\frac{t_{m} l_{m}}{P_{m}} \frac{d_{m}^{\alpha l_{m} t_{m}} \mathrm{~A}(m)}{P_{m}^{t_{m} l_{m}}}+\lambda l_{m}=0 \\
\frac{\partial \Gamma}{\partial d_{m}}=\frac{\alpha t_{m} l_{m}}{d_{m}} \frac{d_{m}^{\alpha l_{m} t_{m}} \mathrm{~A}(m)}{P_{m}^{t_{m} l_{m}}}+\mu=0
\end{gathered}
$$

The relationship of $P_{m}$ and $d_{m}$ for $m=1,2, \ldots, M$ can be written as: $P_{m}=-\mu d_{m} /\left(\alpha \lambda l_{m}\right)$. Accordingly, we have $P_{T}=-\mu / \alpha \lambda l_{m}$ and $P_{m}=d_{m} P_{T}$. By substituting them into (11) and (12), we have: $P_{m}^{*}=\left(\frac{\lambda^{*} P_{T}^{\alpha l_{m} t_{m}}}{t_{m} \mathrm{~A}(m)}\right)^{\frac{1}{(\alpha-1) l_{m} t_{m}-1}}$ and $d_{m}^{*}=\left(\frac{\lambda^{*} P_{T}^{t_{m} l_{m}+1}}{t_{m} \mathrm{~A}(m)}\right)^{\frac{1}{(\alpha-1) l_{m} t_{m}-1}}$, where $\lambda^{*}$ satisfies $\sum_{m=1}^{M}\left(\frac{\lambda^{*} P_{T}^{t_{m} l_{m}+1}}{t_{m} \mathrm{~A}(m)}\right)^{\frac{1}{(\alpha-1) l_{m} t_{m}-1}}=D$.

Note that the solution of the joint optimization is a special case of a separate optimization resulting in the best performance. Apparently, the joint optimization of PA and RP provides the best performance.

The mathematical analysis shows that the e2e outage probability performance of the proposed joint optimization scheme is related to the number of transmissions in relays. Unfortunately, a closed-form expression for $l_{m}$ appears to be hard to find. In this paper, we shed some light on the performance analysis by proposing a retransmission allocation strategy based on the joint OPA and ORP strategy. The retransmission allocation strategy is done in two steps. In the first step, we set retransmission number as $l_{m}=\lfloor L / M\rfloor, \mathrm{m}=1,2, \ldots \mathrm{M}$. In the second step, if $L$ is not a multiple of $M$, use the retransmission time on the weak link. Otherwise, we allocate one retransmission chance from the strongest hop to the weakest hop. As a consequence, more retransmission chance is allocated to the weaker hop to leverage the performance of weaker hops. Since the channel difference can be compensated by the joint optimization, the proposed scheme achieves equivalent or better e2e outage probability.

\section{Analytical and simulation results}

In this section, the simulation and analytical results are presented to verify the performance of optimized multihop relay networks in various fading channels. The performance is evaluated with normalized distance, three hop $(M=3)$ relay networks with pathloss exponent $\alpha=3$, and SNR threshold $\gamma_{t h}=5 d B$, unless stated otherwise. The total SNR constraint is denoted by $\rho_{T}=P_{T} / N_{0}$. The analysis results are compared with the Monte-Carlo simulation results. In the following figures the analysis results are denoted with lines while the simulation results are denoted with marks. 


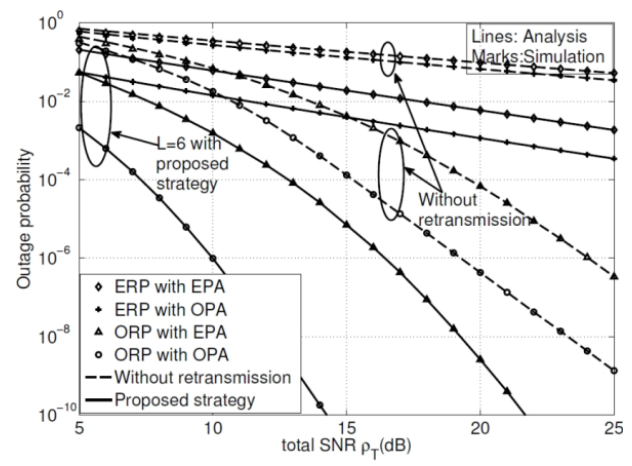

Figure 1. The $P_{e 2 e}$ of three hop relay networks versus total SNR $\rho_{T}$ in Nakagami-m fading channels with $(m 1, m 2, m 3)=(0.5,1,5)$.

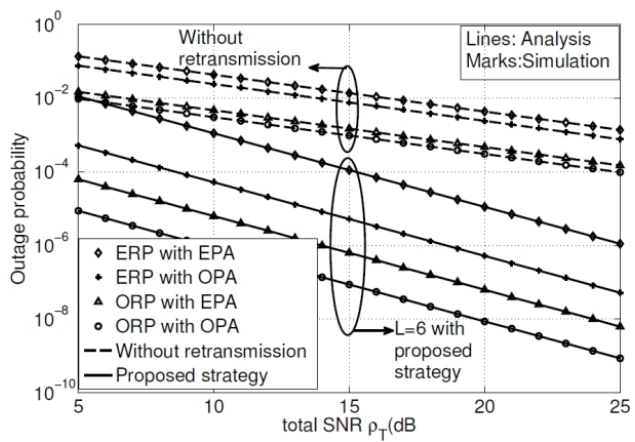

Figure 2. The $P_{e 2 e}$ of three hop relay networks versus total SNR $\rho_{T}$ in Rician fading channels with $(K 1, K 2, K 3)=(1,5,7)$.

Fig. 1 and Fig. 2 compare the e2e outage probability $P_{e 2 e}$ of three hop relay networks versus SNR $\rho_{T}$ in unbalanced channel parameters for Nakagami fading channel with $(m 1, m 2, m 3)=(0.5,1,5)$ and Rician fading channels with $(K 1, K 2, K 3)=(1,5,7)$. It can be observed that the analytical results match the simulation results nicely for a wide range of SNR, which verify the accuracy of the algorithm. Although ORP with equal PA (EPA) scheme outperform OPA with equal RP (ERP) schemes, the separate optimization of PA and RP are suboptimal to the joint optimization of PA and RP as expected. It should be noticed that the performance gains come form the compensation of the channel difference. In the meantime, the HARQ schemes with retransmission number $L=6$ are compared with the scheme without retransmission. According to the results, the outage probability is significantly improved by the use of HARQ techniques. An important observation is that the joint optimization scheme is more sensitive to retransmission. This implies that the gain from optimization gets better as the channel condition gets more favourable.

To evaluate the effect of retransmission on different RP and PA strategy, the $P_{e 2 e}$ of three hop relay networks versus retransmission number $L$ in Nakagami-m fading channel with $(m 1, m 2, m 3)=(1,1,5)$ and total SNR $\rho_{T}=10$ are shown in Fig.3. Three power and relay optimization strategies are compared for two retransmission strategies, i.e., the average retransmission strategy which is denoted by the dash-dot lines and the proposed retransmission strategy which is denoted by soiled lines. For comparison, we also include in the figures the transmission power level of the equal- 
retransmission assignment strategy. We observe that the proposed retransmission strategy achieves significant performance improvement within the first few retransmission rounds. The proposed retransmission strategy shows better performance compared to the equal-retransmission strategy. The reason is that with the increase of the total power, the outage probability of the hops with better channel condition will decrease much faster than that of the weaker hop due to the channel difference. By allocating more retransmission chance to the weaker hop, the proposed retransmission strategy eventually improves the e2e performance. Moreover, the e2e outage performance improves with increasing number of retransmission numbers and the performance gains in the outage probability do not increase linearly.

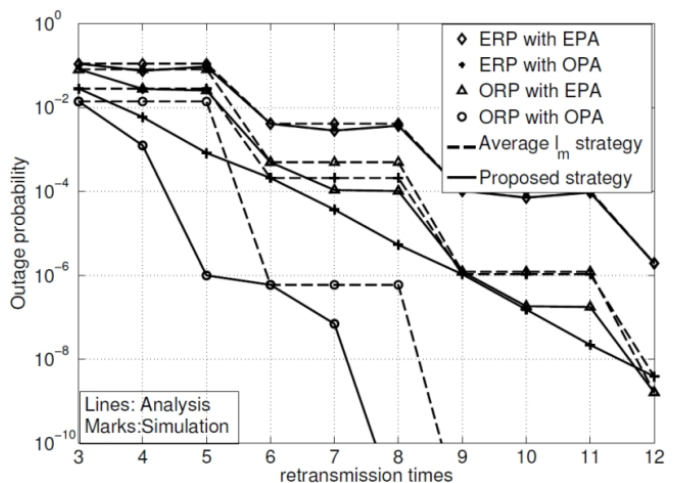

Figure 3. The $P_{e 2 e}$ of three hop relay networks versus retransmission number $L$ in Nakagami- $m$ fading channel with $(m 1, m 2, m 3)=(1,1,5)$ and total SNR $\rho_{T}=10$.

\section{Conclusion}

This paper has addressed the problem of joint optimization of OPA and ORP of a multihop relay networks adopting HARQ. The expression for the e2e outage probability is derived under generalized fading channels and optimization is carried out to find joint optimal power allocation (OPA) and relay placement optimal (ORP) for multihop relay networks. The result demonstrates that the equidistant relay placement with equal power allocation strategy is not the optimal way of power apportionment for unbalanced channel conditions, and the proposed strategy can improve the e2e outage probability significantly by dramatic compensating the channel difference. As future work, the proposed policy can be extended to systems employing practical coding and modulation schemes. Furthermore it is interesting to consider the range expansion and power allocation problem when the end-to-end retransmission constraint is enforced on the whole HARQ process.

\section{References}

1. K. Dhaka, R. K. Mallik, R. Schober. : 'Optimisation of power allocation for asymmetric relay placement in multi-hop relay systems ', IET Commun., 7, 2, pp. 128-136.(2013).

2. Caleb K. Lo, S. Vishwanath, and R. W. Heath, Jr. `An Energy-Based Comparison of Long-Hop and Short-Hop Routing in MIMO Networks', IEEE Trans. Veh. Technol., 59, 1, pp. 394-405 (2010).

3. X. Chen, S. H. Song and K. B. Letaief. : 'Relay Position Optimization Improves Finite-SNR Diversity Gain of Decode-and-Forward MIMO Relay Systems', IEEE Trans. Commun., 60, 11, pp. 3311-3321, (2012).

4. S. H. Kim, S. J. Lee, and D. K. Sung. 'HARQ Rate Selection Schemes in a Multihop Relay Network With a Delay Constraint ', IEEE Trans. Veh. Technol., 64, 6, pp. 2333-2348, (2015). 
5. A. A. Haghighi and K. Navaie. : 'Truncated HARQ-Based Multi-Hop Systems:Outage and Related Performance Metrics ', IEEE Trans. Wireless Commun., 63, 10, pp. 303-313, ( 2015).

6. M. K. Simon, M. S. Alouini,: Digital Communication over Fading Channels. (John Wiley and Sons, second edition, 2005.)

7. Z.Wang and G. Giannakis. : 'A simple and general parameterization quantifying performance in fading channels ', IEEE Trans. Commun., 51, 8, pp. 1389-1398, (2003).

8. T. P. Do, I. Song and Y. H. Kim. : 'Optimization of Power Allocation and Relay Position in Multihop Relay Networks Over Generalized Fading Channels ', IEEE Trans.Wireless Commun., 64, 11, pp. 5031-5041, (2015).

9. GGPLAB: A Simple MATLAB Toolbox for Geometric Programming. Available: http://www.stanford.edu/ boyd/ggplab/. 